\title{
Multiband OFDM for Cognitive Radio - A Way for Cyclostationary Detection and Interference Cancellation
}

\author{
J. Avila, K. Thenmozhi
}

Department of Electronics and Communication Engineering, SASTRA University, Thanjavur, Tamil Nadu, India

\begin{tabular}{l}
\hline \hline Article Info \\
\hline Article history: \\
Received Feb 17, 2015 \\
Revised May 17, 2016 \\
Accepted May 30, 2016 \\
\hline
\end{tabular}

\section{Keyword:}

Cognitive radio Improved AIC Multiband OFDM Spectrum sensing USRP

\begin{abstract}
With the tremendous growth in wireless technology there has been a shortage in the spectrum utilized for certain applications while some spectrum remains idle. To overcome this problem and for the efficient utilization of the spectrum cognitive radio is the suitable solution. Multiband OFDM can be easily modeled as cognitive radio, a technology that is employed for utilizing the available spectrum in the most efficient way. Since sensing of the free spectrum for detecting the arrival of the primary users is the foremost job of cognitive, here cyclostationary based spectrum sensing is carried out. Its performance is investigated using universal software defined radio peripheral (USRP) kit which is the hardware test bed for the cognitive radio system. Results are shown using Labview software. Further to mitigate the interference between the primary and cognitive users a modified intrusion elimination (AIC) algorithm had been proposed which in turn ensures the coexistence of both the users in the same wireless environment.
\end{abstract}

Copyright (c) 2016 Institute of Advanced Engineering and Science. All rights reserved.

\section{Corresponding Author:}

Avila J,

Department of Electronics and Communication Engineering,

SASTRA University,

Thanjavur, Tamil Nadu- 613401, India.

Email: avila@ece.sastra.edu

\section{INTRODUCTION}

A primary protocol of UWB communication is the Multiband OFDM (MB-OFDM) system that supports high data rates while consuming very less power [1]. It is preferred because it addresses the multipath fading problem thereby making it a good communication system in an urban/indoor area. Hence further discussion for improvement of its performance in a high congestion, high noise environment is imperative. The best way to improve the performance without changing the trans reception protocol is by the use of Error control codes [2]. While high speed data rates are of the norm of the day, this demand inadvertently leads to the higher error rates. To tackle it concatenation of error control codes is performed [3] for they have a higher coding gain than a single code.

It becomes necessary that the multiband OFDM system [4] must coexist with various well established protocols such as radio Astronomy of Japan, WiMax devices, IEEE 802.11 devices and so on. Hence interference cancellation between the multiband OFDM system and other becomes a foremost task. The development of wireless communication as a viable source of income led to the advent of licensed spectrums. The downside of this was the inefficient use of allotted spectrum, which is now being addressed. by the development of Cognitive radio [5]. Since in the multiband OFDM system the Fast Fourier Transform operation is performed at the receiver side, it can be easily modelled as a cognitive radio .They address the problem by scanning and sensing the unused spectrum in the licensed area and introducing a secondary user to occupy it [6] The simplest way of sensing the used and unused spectrum is by the use of cyclostationary method, which compares the energy of the signal to a predefined value and states whether that spectrum is occupied or not [7],[8]. This paper mainly focuses on the performance of this cyclostationary method of 
sensing [9]. Since the UWB system incorporated data over a wide range of frequencies, it is imperative that the interference between the primary user and the cognitive user were limited to a minimum.

The word LabVIEW is an abbreviation for Laboratory Virtual Instrumentation Electronic Workbench. This software uses G-language (Graphical User Interface language) .It helps in virtually simulating any of the instruments functioning without actually having to use the hardware components of the circuit. The VI's are the programs which represent the functioning of the instrument. Each vi has two windows named the front panel and block panel. The front panel is the representation of the instrument i.e. how it will look after practically being implementing the idea. It has all the controlling knobs the instrument is expected to have and the in simple words it's the interface for the user. The block panel has the full functions of the VI. That is it has the functions which perform the action of the instrument [10].

This work concentrates on the interference between the multiband OFDM acting as cognitive radio and other wireless systems that coexist in the same environment. Being a cognitive radio it performs spectrum sensing (ie) the most vital job of any cognitive radio is to sense the arrival of licensed users and availability of the free spectrum. Cyclostationary based spectrum sensing is discussed. The hardware test bed for the cognitive radio is setup with the use of Universal software define peripheral kit with Labview as the processing tool.

Next part explains in detail about the proposed methodology. The last part deals with the discussion of results and finally this research contribution has the concluding remarks on the proposed system.

\section{RESEARCH METHOD}

The proposed work consists of two stages.

- Cyclostationary based spectrum sensing to find the existence of primary user

- Effective interference cancellation between the primary user and cognitive user

The block diagram of the LabVIEW based cyclostationary method is shown in Figure 1. It comprises of FFT block, followed by windowing, correlating and magnitude squaring. The output of these blocks yields the power spectral density. The output is then compared with the predefined threshold. If the received signal crosses the threshold the primary user is present and vice versa. The decision is made on the hypotheses

$\mathrm{H}_{0}=$ signal+ noise : Primary user present

$\mathrm{H}_{1}=$ noise : primary user absent

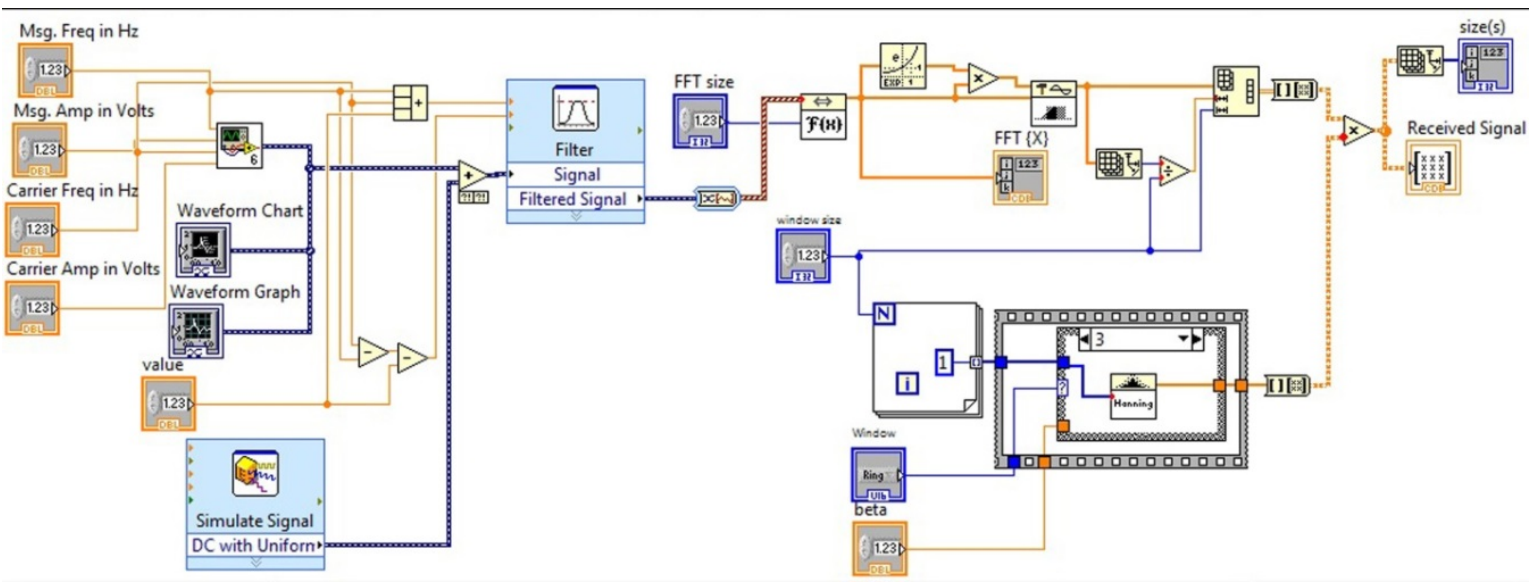

Figure 1. Block diagram of cyclostationary method (Labview)

The proposed block diagram for interference cancellation is shown in Figure 2. The BPSK modulated data is grouped. The subcarriers are grouped in three ways. Adjacent, Interleaved and random partitions are carried out in this work. In adjacent partition the subcarriers which are adjacent to each other are grouped. In interleaved partition alternate subcarriers are grouped .In random partition the subcarriers are chosen and grouped in a random manner. Out of 128 sub-tones it is assumed that three tones namely 85, 86 and 87 as interfering tones. Since there are 128 sub-tones the number of grouping here is four with 32 
subcarriers in each group. The output of partitioning block is given as input to AIC block. The MIMO AIC algorithm is given by the transmitted signal is written in matrix form as $E=n m$, where

$$
\begin{aligned}
& n=[n(0) n(1) \ldots \ldots \ldots \ldots \ldots \ldots(i-1)]^{T} \\
& m=[m(0) m(1) \ldots \ldots \ldots \ldots \ldots . . . . m(s \times i-1)]^{T} \\
& E=\left[\begin{array}{l}
E_{11} \ldots \ldots \ldots \ldots E_{1 i} \\
\cdot \\
\cdot \\
E_{s i, 1} \cdots \cdots \cdots E_{s i, i}
\end{array}\right] \\
& E_{k i}=E(k, l)=\sum_{m=0}^{N-1} \exp \left(j 2 \pi \frac{m}{N}\left(i-\frac{k}{s}\right)\right) \\
& c=\arg _{c} \min \left\|E_{1} c+v_{1}\right\|^{2}
\end{aligned}
$$

$\mathrm{C}$ is the target AIC vector. The interference vector is given by

$$
v_{1}=v\left(f r_{1}+y: f\left(r_{n}-1\right)-(y+2)\right), y \geq 0
$$

The minimization problem is given by

$$
\begin{aligned}
& \min _{x}\left\|E_{x}-F\right\|^{2} \quad \text { subject to }:\left\|G_{x}-H\right\|^{2}<\alpha \\
& C_{\text {opt }}=\arg _{c} \min \left\|E_{1} c+v_{1}\right\|^{2}
\end{aligned}
$$

Subject to: $\quad\left\{\quad\left|C_{E T .1}(k)\right|^{2} \leq \mathcal{E}_{i} ; i=1, \ldots . . N_{E T} / 2\right.$

$$
\left|C_{E T .2}(k)\right|^{2} \leq \varepsilon_{j} ; j=1, \ldots . . N_{E T} / 2
$$

$$
C=\left[\boldsymbol{C}_{E T .1} \boldsymbol{C}_{\text {null }} \boldsymbol{C}_{E T .2}\right]^{2}
$$

Lagrange optimization algorithm is used to optimize the problem which is given as

$$
\begin{aligned}
& f\left(c, \lambda_{i}, \lambda_{j}\right)=\mid E_{1} c+V_{1} \|^{2}+\sum_{i=1}^{N_{E T}^{\prime 2}} \lambda_{i}\left(\left|C_{E T .1}(i)\right|^{2}-\varepsilon_{i}\right)+\sum_{j=1}^{N_{E T}^{\prime 2}} \lambda_{j}\left(\left|C_{E T .2}(j)\right|^{2}-\varepsilon_{j}\right) \\
& \left(E_{1}^{H} E_{1}+\Lambda\right) C_{o p t}=E_{1}^{H} V_{1} \\
& C_{o p t}=-\left(E_{1}^{H} E_{1}+\Lambda\right)^{-1} E_{1}^{H} V_{1} \\
& \Lambda=\operatorname{diag}\left[\lambda_{1} \cdots \cdots \cdots \cdot \lambda_{\alpha} 0 \ldots \ldots . .0 \lambda_{\beta} \cdots \cdots \cdots \lambda_{N_{E T}}\right]
\end{aligned}
$$




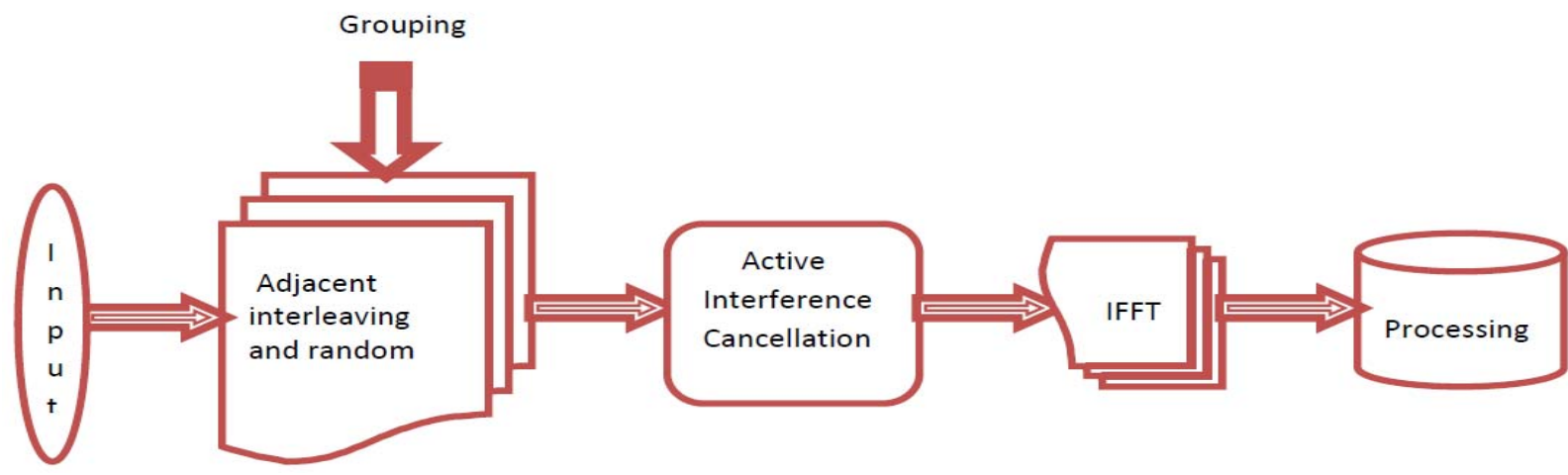

Figure 2. Block diagram of proposed MIMO AIC

\section{RESULTS AND ANALYSIS}

Single input single output (SISO) based Active interference algorithm was proposed in [11] which was further enhanced in by cyclic shifting and phase shifting of the data [12]. Statistical relations based Active interference canceller (AIC) was proposed in [13] to improve the basic AIC algorithm. The use of partial transmit sequence mitigation is carried out in [14].Introduction of weighting factors on several subcarriers mitigation is carried out inv [15]. Multi input multi output (MIMO) based active interference cancellation was proposed in [16], which was further enhanced in [17]. The main focus of this paper is to propose a modified intrusion elimination technique for MIMO based multiband OFDM cognitive radio system.

The first two results are plotted using MATLAB tool. The performance metric is subcarrier index versus magnitude spectrum. The remaining results are plotted using LabVIEW tool. Here the performance metric is Probability of detection ( $P_{d}$ ) versus Signal to Noise Ratio (SNR).

Figure 3 shows the adjacent partition without weighting factors. The 128 subcarriers are grouped into 4 groups. Adjacent carriers are grouped. Then the modified AIC algorithm is applied. Figure 4 shows the output of adjacent partition with weighting factors. From the figure it is clear that adjacent partition with weighting factors dominates. A deep notch is achieved. There is at least $1 \mathrm{~dB}$ difference between both the cases.
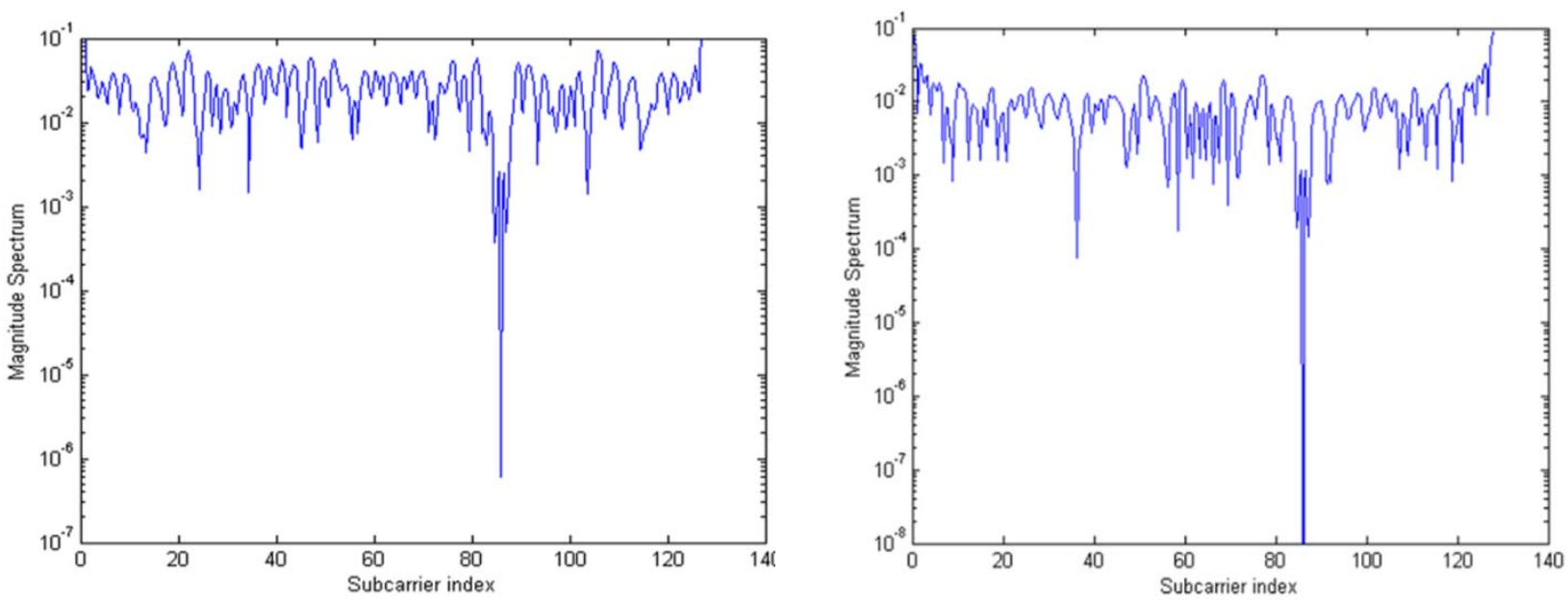

Figure 3 and 4. Adjacent partition with and without weighting factors

Figure 5 shows the random partition without weighting factors. The 128 subcarriers are grouped randomly. Then the modified AIC algorithm is applied. Figure 6 shows the random partition with weighting factors. The 128 subcarriers are grouped randomly. Then the weighting factors are applied. The weighting factors are based on the modulation scheme used. Since in this work it is BPSK modulation the weighting 
factors are chosen as +1 and -1 . After applying the weighting factors AIC algorithm is used. From the figures it is clear that a deep notch is achieved with the inclusion of weighting factors. Deep notch in turn symbolizes that the interference between the primary user and the cognitive used is reduced.
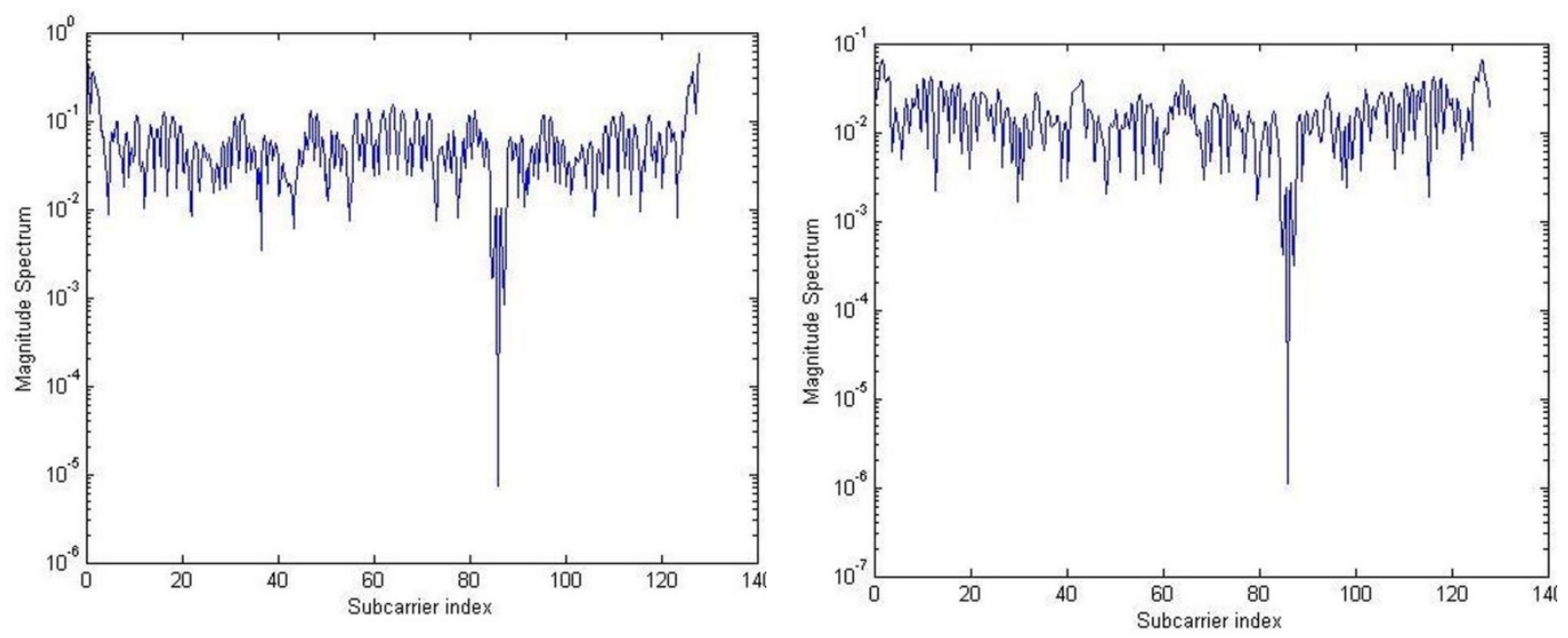

Figure 5 and 6. Random partition with and without weighting factors

Figure 7 shows the hardware setup for the cyclostationary based spectrum sensing. Here two USRPs are used. One acts as transmitter and the other acts as receiver. USRP is a transceiver which converts a personal computer into wireless prototype system. The basic components of the USRP are RF amplifier, Local oscillator, ADC and DAC. The architecture also includes FPGA for processing the data. USRP 2920 has been utilized in this work. The USRPs were connected to their host personal computers through 2.0 Ethernet cable. The IP address of the transmitter was set as 192.168.10.1 and that of receiver was set as 192.168.10.2. At the receiver side, the cyclostationary based spectrum sensing module developed using LabVIEW was utilized and detection of PU was done and plotted.

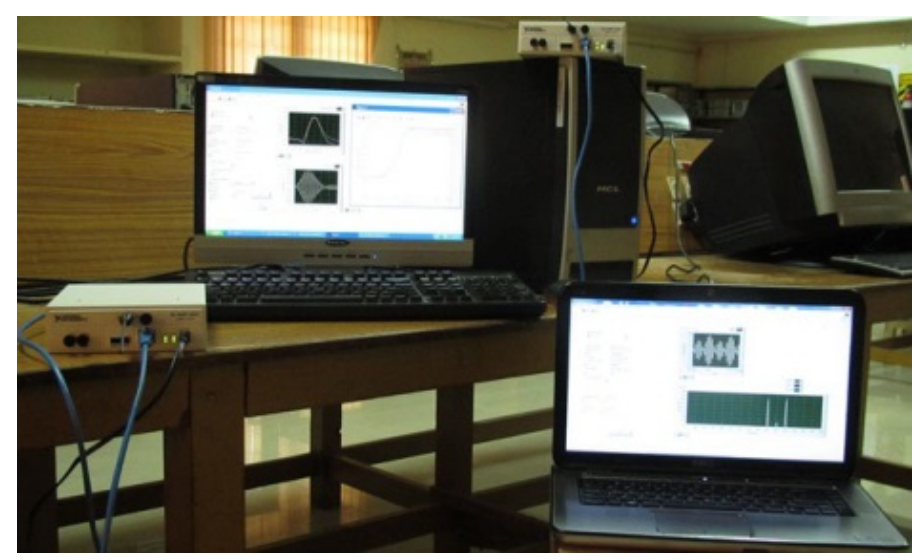

Figure 7. Hardware setup

Figure 8 gives the comparison between various FFT sizes (128,256,512 and 1024) in cyclostationary method. Hamming window with window size of 10 is used. The graph shows that with increase in FFT size probability of detection could be achieved earlier. For the Probability of detection $\left(P_{d}\right)$ of 0.9 the SNR for the FFT size of 1024 is $-27 \mathrm{~dB}$ whereas the SNR value is $-22 \mathrm{~dB}$ for the FFT size of 128 . As the FFT size increases frequency resolution increases and it helps in detecting the primary used in high noisy environment. 


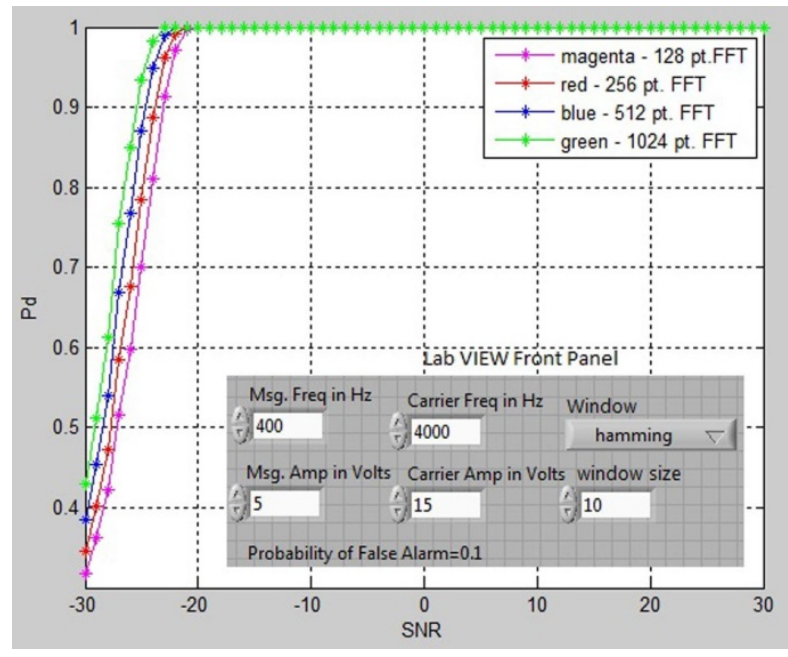

Figure 8. various FFT sizes

Figure 9 gives the comparison between various window sizes in cyclostationary method. Hamming window with FFT size of 512 is used. For the Proabability of detection $\left(P_{d}\right)$ of 0.9 the SNR value is $-25 \mathrm{~dB}$ for the window size of 10 whereas for the same $P_{d}$ the SNR value is $-27 \mathrm{~dB}$ for the window size of 40 . The graph shows that with increase in window size the resolution increases and hence the primary users could be detected in low SNR condition.

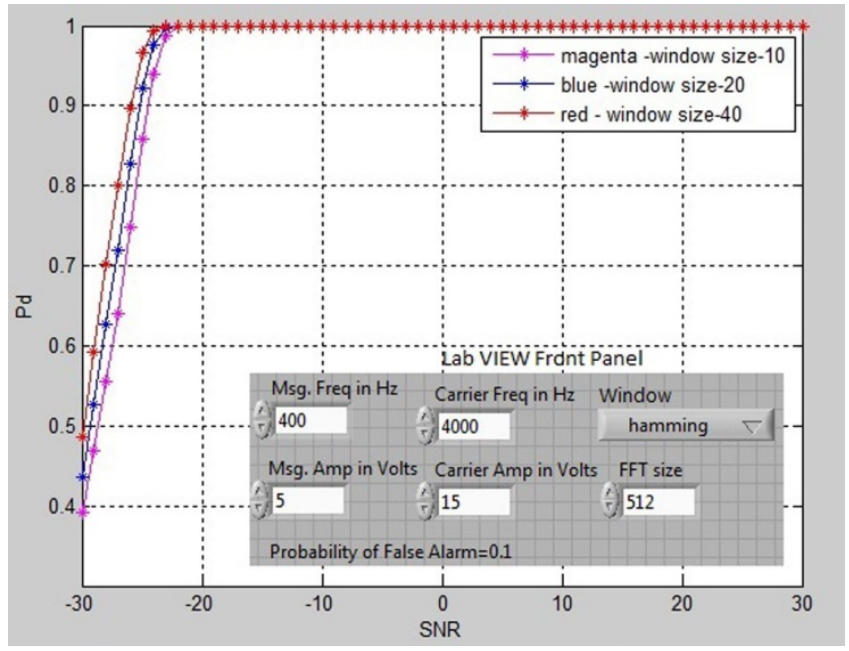

Figure 9. various window sizes

Figure 10 gives the comparison between various windows in cyclostationary method. Hamming window, Blackman window and Kaiser Window are compared. The graph shows Kaiser Window offers better $P_{d}$ when compared with other windows. For the Probability of detection $\left(P_{d}\right)$ of 0.9 the SNR value is $-25 \mathrm{~dB}$ in case of Kaiser window whereas it is $-23 \mathrm{~dB}$ for Blackman window. It is governed by the parameter beta which helps in controlling the width of the main lobe. Greater the main lobe and lesser the side lobe depicts that the spectral leakage is less. Hence proper choice of the window enhances the system performance. 


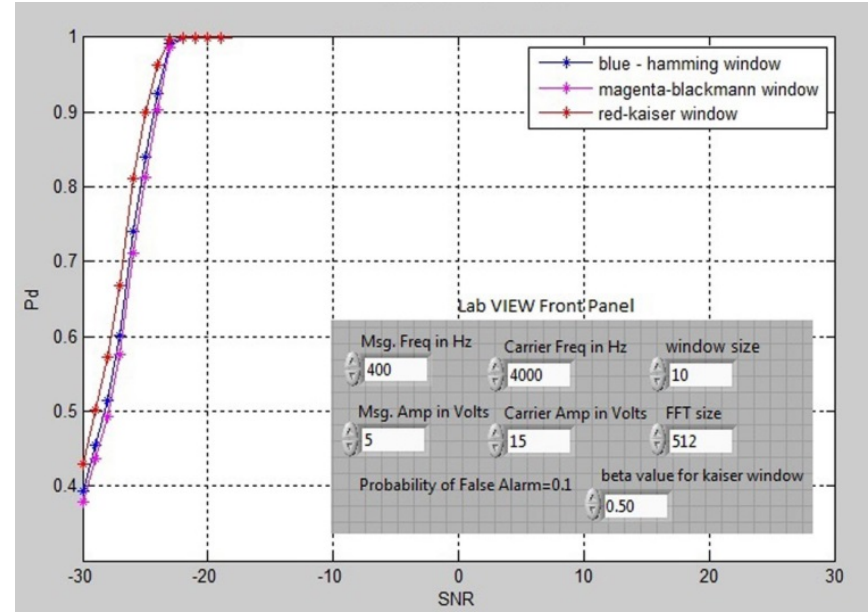

Figure 10.various windows

Figure 11 gives the comparison between various values of Probability of false alarm $\left(P_{f}\right)$ in cyclostationary method. For the Probability of detection $\left(P_{d}\right)$ of 0.7 the SNR value is -28dB for the $P_{f}$ of 0.1 whereas the SNR value is $-26 \mathrm{~dB}$ for the $P_{f}$ value of 0.0001 . Figure shows lesser the $P_{f}$ value error decreases and probability of detection is accomplished in high noisy environment.

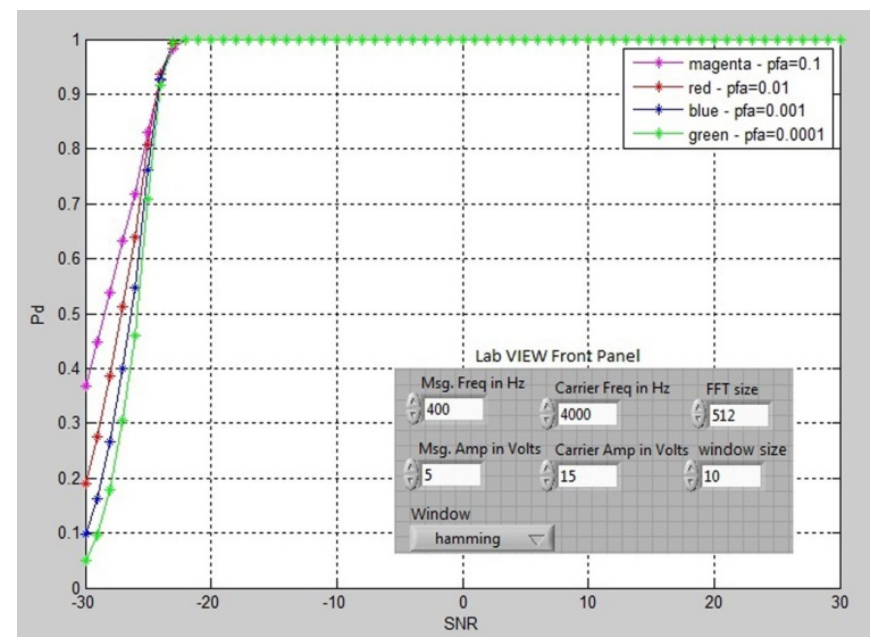

Figure 11.various probability of false alarm

\section{CONCLUSION}

Cognitive being a solution for efficient utilization of the sparse spectrum, the performance of cyclostationary based spectrum sensing method is analyzed. To mitigate the interference between primary user and secondary user a modified interference reduction algorithm has been proposed and it is seen that there is an improvement in notch depth with the insertion of weighting facotrs. All these techniques enhance the performance of the multiband OFDM system and makes it more appropriate and a perfect solution when the noise is so severe that the juice of high data rates can be enjoyed.

\section{ACKNOWLEDGEMENTS}

The Authors would like to express their sincere thanks to SASTRA University, for the grant received under R\&M fund(R\&M / 0027 / SEEE - 010 / 2012 - 13) to carry out this research work. 


\section{REFERENCES}

[1] J. Balakrishnan, et al., "A multiband OFDM system for UWB communication," in IEEE conference on ultrawide band systems and technology, pp. 354-358, 2003. doi: 10.1109/UWBST.2003.1267863.

[2] P. Praveenkumar, et al., "Regulated OFDM-role of ECC and ANN-A review," Journal of Applied sciences, vol. 12, pp. 301-314, 2012. doi: 10.3923/jas.2012.301.314.

[3] J. Avila, et al., “Anti Interference for anti corroupt for 3G spectrum-A multi carrier approach,” in IEEE International conference on computer communication and Informatics, pp. 1-4, 2012.

[4] A. Batra, et al., "Multiband OFDM: A cognitive radio for UWB," in Proceedings of International symposium on circuits and systems, pp. 4093-4097, 2006.

[5] S. Haykin, "Cognitive radio: Brain empowered wireless communications," IEEE Journal on selected areas in communications, vol. 23, pp. 201-220, 2006.

[6] M. Hejazi and B. Abolhassani, "Energy detection based spectrum sensing in cognitive radio networks over spatially-correlated channels,” in IEEE Symposium on Industrial Electronics \& Applications (ISIEA), pp.738-743, 2010. doi:10.1109/ISIEA.2010.5679368.

[7] G. A. F. M. Khalaf, “An optimal Sinsing algorithm for Multibnad Cognitive Radio Network,” International Journal of Information \& Network Security, vol. 2, pp. 60-67, 2013.

[8] W. S. Shan, et al., "Primary User Emulation Attacks Analysis for Cognitive Radio Networks Communication," TELKOMNIKA Indonesion Journal of Electrical Engineering, vol. 11, pp. 3905-3914, 2013.

[9] V. Prithiviraj, et al., "Cyclostationary Analysis Method of Spectrum Sensing for Cognitive Radio," in IEEE International conference on wireless communication, vehicular technology, Information theory and Aerospace \& Electronic system technology, pp. 1-5, 2011. doi: 10.1109/WIRELESSVITAE.2011.5940821.

[10] J. Avila and K. Thenmozhi, "Cognitive (LabVIEW) on FPGA-a novel approach for dynamic spectrum sensing," International Journal of electronic letters, vol. 3, pp. 13-23, 2014. doi:10.1080/21681724.901422

[11] H. Yamaguchi, “Active Interference Cancellation technique for MB-OFDM Cognitive radio," in Proceedings of 34th European Microwave conference, pp. 1105-1108, 2004.

[12] P. Tarasak, et al., "Further Enhancement for Active Interference Cancellation on MB-OFDM UWB Transmission," in IEEE $68^{\text {th }}$ conference on vehicular technology, pp. 1-5, 2008. doi: 10.1109/VETECF.2008.244.

[13] S. G. Huang and C. H. Hwang, "Improvement of Active Interference Cancellation: Avoidance Technique for OFDM Cognitive Radio,” IEEE Transactions on Wireless Communication, vol. 8, pp. 5928-5937, 2009.

[14] P. Tarasak, et al., "Partial Transmit Sequence-Active Interference Cancellation for UWB OFDM Transmission," in IEEE $20^{\text {th }}$ Int. Symposium on personal Indoor and Mobile Radio Communications, pp. 943-947, 2009. doi: 10.1109/PIMRC.2009.5450339.

[15] A. Minakawa, et al., "A study on interference suppression using weighting factors for MB-OFDM systems," in IEEE International Conference on Ultrawide band, pp. 88-92, 2009. doi: 10.1109/ICUWB.2009.5288702.

[16] F. Sarabchi and C. Nerguizian, "Interference cancellation Technique for MIMO MB-OFDM UWB Cognitive Radio System," in 6th International Conference Wireless and Mobile Communications, pp. 472-477, 2010. doi: 10.1109/ICWMC.2010.53.

[17] J. Avila, et al., "ANN assisted-Augumentation of AIC for MIMO Multiband OFDM System,” in IEEE International conference on Advances in Engineering, Science and Management, pp. 228-232, 2012.

\section{BIOGRAPHIES OF AUTHORS}

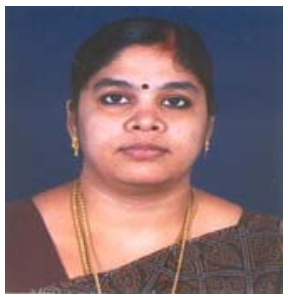

J. Avila received her B.E (ECE) from the V.M.K.V college of Engineering and M.E (Communication Engineering) from Vellore Institute Of Technology. Currently she is working as Assistant Professor III in the Department of ECE in SASTRA University, Thanjavur. She has a teaching experience of 13 years and she has published 19 Research articles in National \& International journals. She is currently working towards her Ph.D. Degree in SASTRA University. Her research area includes Wireless communication and Cognitive radio

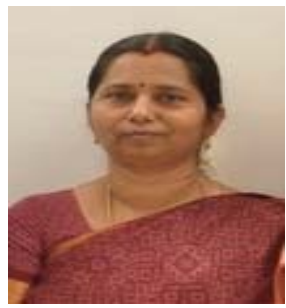

K. Thenmozhi received her B.E (ECE) and M.E (Communication system) degrees from Regional Engineering college (NIT) Tiruchirappalli and Ph.D. from SASTRA University, Thanjavur. Currently she is working as an Associate Dean in the Department of ECE in SASTRA University, Thanjavur. She has a teaching experience of 23 years. Her current research area includes Wireless communication, Steganography and Information Theory and Coding. She has supervised more than 100 UG projects, 10 Master Students and Supervising 5 Ph.D. Scholars. So far she has published 50 Research articles in National \& International journals conferences. She received EDI award from the broadcast Engineering Society for the year 2007. 